\title{
REVIEW
}

\section{Customising chemotherapy in advanced nonsmall cell lung cancer: daily practice and perspectives}

\author{
A.C. Vilmar and J.B. Sorensen
}

ABSTRACT: Treating patients with advanced nonsmall cell lung cancer (NSCLC) is a daunting task but during recent years new options have emerged. By tailoring treatment using either information on histological subtypes of NSCLC or biomarkers it is now possible to improve outcome and maintain stable quality of life.

We conducted a literature search of tailored treatment already implemented in advanced NSCLC in order to highlight the information required to decide on the optimal oncological treatment for individual patients.

16 studies were identified by literature review. Significantly improved outcome was demonstrated in patients with nonsquamous NSCLC treated with cisplatin/pemetrexed in pre-planned, exploratory and retrospective analysis from large-scale, randomised trials. Level 1 evidence showed significantly better progression-free survival when patients carrying an epidermal growth factor receptor (EGFR) mutation were treated with gefitinib compared to standard chemotherapy. Retrospective, unplanned analysis of excision repair cross complementation group 1 (ERCC1) and betatubulin III upregulation demonstrated poorer outcome in NSCLC patients treated with platinum-doublets and vinorelbine-based chemotherapy, respectively.

In conclusion, tailoring treatment according to either histological subtype or EGFR mutation status in advanced NSCLC should today be part of daily practice based on current evidence. Future biomarkers need optimisation of methodology and prospective validation before clinical implementation.

KEYWORDS: Advanced nonsmall cell lung cancer, customised treatment, predictive biomarkers, review


he incidence of lung cancer in Europe in 2008 was estimated to 390,900 cases, and 342,100 patients died of the disease [1]. Throughout the Western world, lung cancer remains the leading cause of cancer-related deaths, as well as being characterised by great morbidity. Tobacco smoke is still the main causal agent. Around $80 \%$ of patients with lung cancer are diagnosed with nonsmall cell lung cancer (NSCLC) and roughly one-third of these receive curatively intended treatment, leaving the majority candidates for palliative chemotherapy.

Platinum-based doublets (PBDs) remain the cornerstone of chemotherapy in advanced NSCLC and platins combined with either vinorelbine, gemcitabine, paclitaxel or docetaxel are extensively used. Carboplatin-based combinations versus cisplatinbased combinations have resulted in slightly different response rates (RRs), favouring cisplatin combinations ( $24 \%$ versus $30 \%$; $\mathrm{p}=0.001)$, but with largely similar median survival (8.4 months versus 9.1 months) and $1 \mathrm{yr}$ survival rates (34\% versus $37 \%$ ) [2]. Cisplatin is associated with more severe side-effects.

Based on the expanding knowledge in molecular tumour biology, subcellular targets have now been identified as being involved in treatment sensitivity. Thus, a more individualised approach to NSCLC patients may be achieved and outcome may be improved in selected subgroups based on histological subtype $[3,4]$ or biomarkers, such as epidermal growth factor receptor (EGFR) mutations [5-7].

The concept of tailoring treatment by the use of individual biomarker profile is becoming daily
AFFILIATIONS

Dept of Oncology, Finsen Centre, National University Hospital, Copenhagen, Denmark.

CORRESPONDENCE A.C. Vilmar

Dept of Oncology 5073

Finsen Centre

National University Hospital

9 Blegdamsvej

DK-2100 Copenhagen

Denmark

E-mail: adamvilmar@hotmail.com

Received:

Aug 052010

Accepted after revision:

Sept 192010

PROVENANCE

Submitted article, peer reviewed. 
practice in patients with NSCLC, in order to improve outcome and maintain a tolerable quality of life (QoL). Histology-driven treatment decisions have already been applied owing to the results of pre-specified exploratory analyses in large-scale prospective randomised clinical trials (RCTs) [3, 4].

However, the prognosis remains poor, despite these improvements, and in patients with advanced NSCLC life expectancy rarely exceeds $1 \mathrm{yr}$, a statistic that demands further improvement.

This improvement is likely to be achieved through further customisation of treatment as the increasing body of cell biological knowledge is being translated to bedside clinical practice. Extensive research concerning predictive biomarkers (the likelihood of responding to a specific type of treatment) is being carried out and has shown promising results, e.g. in enzymes involved in DNA repair [8-10] and microtubulin dynamics [11].

This paper provides an overview of current evidence concerning the predictive factors necessary to customise oncological treatment in advanced NSCLC. Additionally, examples of research in potential candidate biomarkers are discussed. Thus, this paper provides recommendations on the current situation for clinical use of predictive biomarkers in advanced NSCLC today. It is beyond the scope of this review to discuss all potential biomarkers evaluated in NSCLC, among which the majority is either without significant impact or are not yet completely evaluated, and hence not part of current clinical practice.

\section{MATERIALS AND METHODS}

We performed three separated literature searches using the following key words: 1) advanced NSCLC and histopathology and chemotherapy; 2) advanced NSCLC and EGFR and predictive biomarkers; and 3) advanced NSCLC and ERCC1/ RRM1/betatubulin III/KRAS/predictive biomarkers and chemotherapy. The search was limited from January 1996 through February 2010 among adults only ( $\geqslant 19$ yrs). Only English language abstracts were considered. PubMed was used as the main medium. The selection of studies was based on summary review and then, if found relevant, the full article was retrieved. In addition, these papers were screened in order to disclose other relevant articles. If the full paper was not available (poster presentation, etc.) the reported results are based on the abstract. Furthermore, the abstracts of the American Society of Clinical Oncology (ASCO) and the European Society for Medical Oncology (ESMO) meetings within the past 5 yrs were also considered. Due to the extensive body of literature on this subject and the limitations of this review we decided only to include publications with a representative number of patients (arbitrarily chosen as $n>50$ ) in order to obtain valid results.

Level of evidence and number of patients are included in the tables. The end-points recorded were RR, progression-free survival (PFS), overall survival (OS) and hazard ratios (HRs) when available. The studies were grouped into four tables according to subject of investigation.

\section{RESULTS}

\section{Literature selection}

40 potential abstracts were reviewed and five identified as relevant in search one, regarding publications supporting current use of tailored chemotherapy according to histology.
In search two, 39 potential abstracts were reviewed and six were selected concerning evidence supporting current use of customised chemotherapy according to EGFR status. Regarding evidence supporting potential use of customised chemotherapy according to selected biomarkers (search three) the following number of abstracts were reviewed and selected: excision repair cross complementation group 1 (ERCC1): 39 and two; RRM1: 11 and one; betatubulin III: 20 and one; and KRAS: 12 and one, respectively.

\section{Tailored treatment according to histology}

A summary of the studies using treatment tailored according to histology is provided in table 1 . Unplanned retrospective results from a noninferiority trial, comparing docetaxel with pemetrexed in second-line treatment, demonstrated improved survival in patients with squamous cell carcinomas treated with docetaxel but poorer outcome in patients with nonsquamous histology [14]. Pemetrexed combined with cisplatin and pemetrexed as maintenance treatment have later resulted in statistically significant improved survival in patients with nonsquamous histology in pre-specified exploratory analyses from RCTs [3, 4]. Our group demonstrated, in 2009, results from an unplanned analysis in a RCT comparing triplet chemotherapy (cisplatin, paclitaxel and gemcitabine) with standard doublet chemotherapy (cisplatin and vinorelbine), showing significantly improved PFS and OS in the subgroup of patients with squamous cell carcinoma treated with the triplet combination [12]. Patients with adenocarcinomas treated with bevacizumab plus paclitaxel and carboplatin resulted in significantly improved outcome compared to the group receiving standard chemotherapy alone [13].

\section{EGFR status-driven treatment}

A summary of the studies using treatment driven by patient EGFR status is provided in tables 2, 3 and 4. DouILLARD et al. [16] randomised patients to either gefitinib or docetaxel in a second-line setting and demonstrated improved $R R$ in the gefitinib group with high EGFR gene copy number. The study also revealed that patients positive for EGFR mutation showed significantly improved RR and PFS when treated with gefitinib. Prior to this, MoK et al. [7] explored pre-planned subgroup outcome in Asian non- or light smoking NSCLC patients with adenocarcinomas randomised to either gefitinib or carboplatin/paclitaxel. A significantly better RR and PFS were demonstrated in patients with EGFR mutation and receiving gefitinib, whereas the wild-type patients responded significantly better to standard treatment. The two Japanese groups (MITSUDOMI et al. [6] and INOUE et al. [5]) randomised EGFR mutationpositive patients to either gefitinib or PBDs and demonstrated significantly improved outcome in the tyrosine kinase inhibitor (TKI) groups.

\section{Potential biomarkers in future customisation of treatment}

A summary of the studies investigating the potential use of tailored chemotherapy according to selected biomarkers in advanced NSCLC is given in table 5. Сово et al. [19] found a significant difference in RR in the low ERCC1 genotype arm in patients receiving chemotherapy stratified according to ERCC1-mRNA expression. The difference did not translate to improved PFS or OS. Our group has recently, in an unplanned exploratory analysis of an RCT, shown statistically significant 


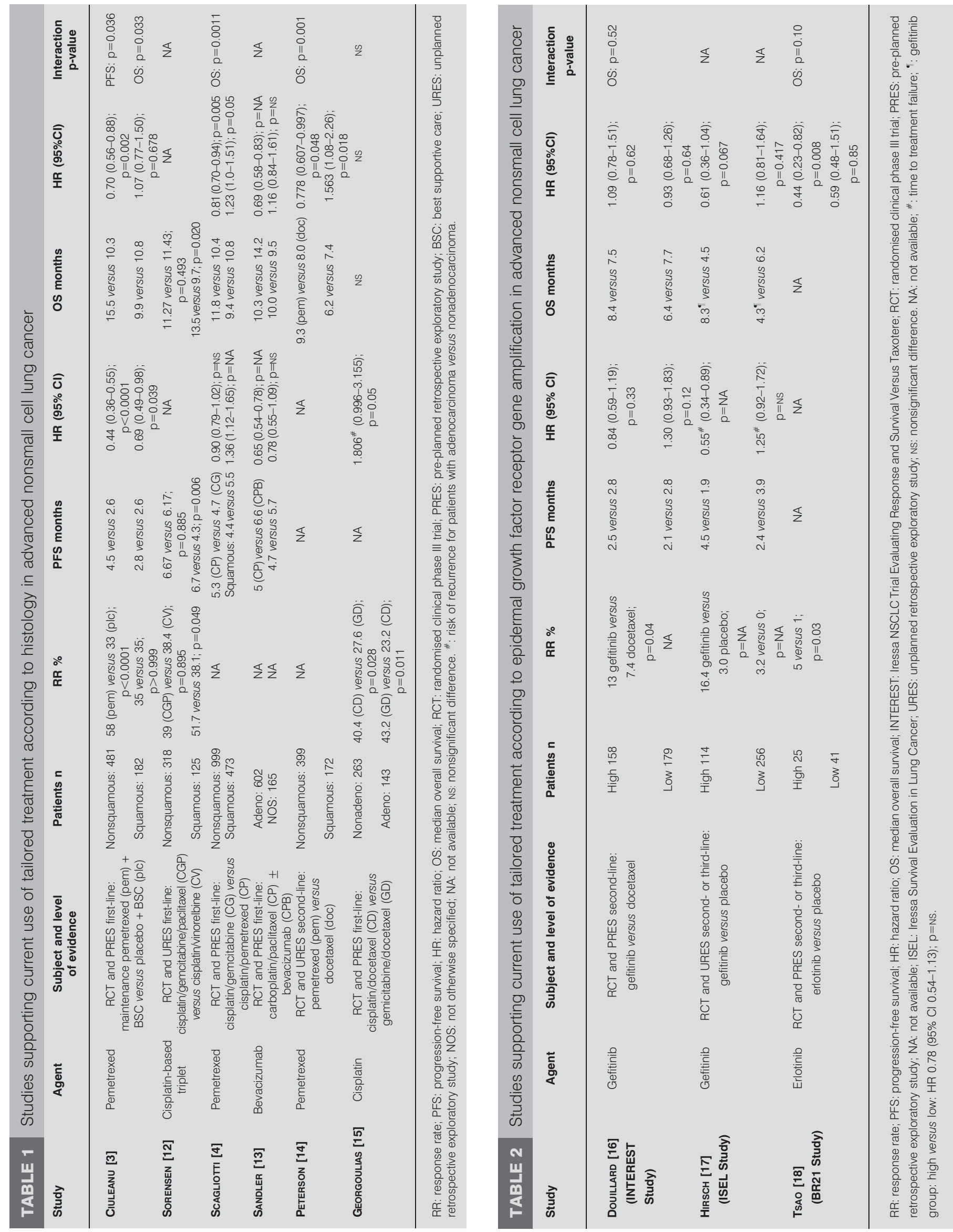




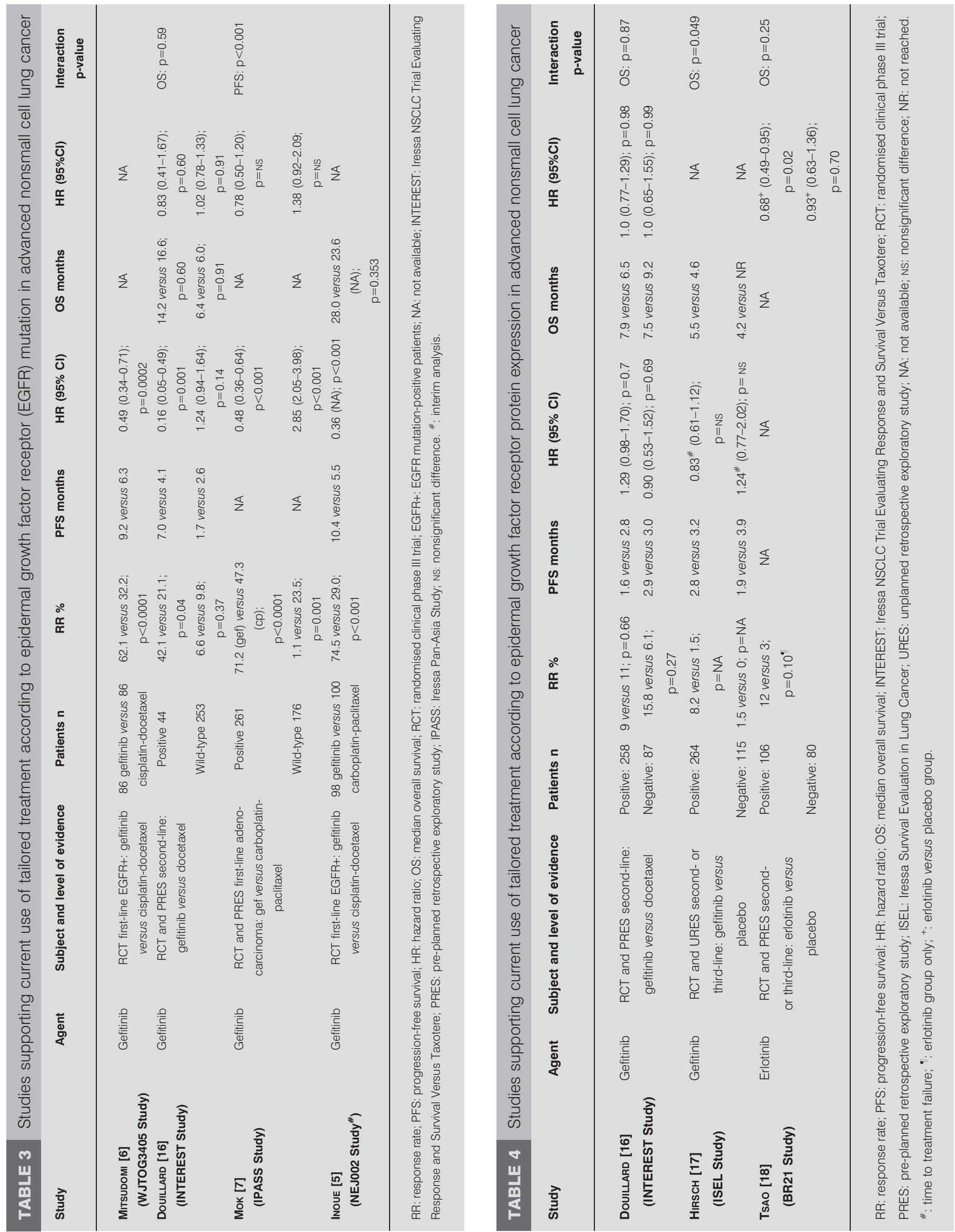






improved outcome in low ERCC1 protein-expressing patients receiving platin-based chemotherapy, as well as interaction between this subgroup and patients with adenocarcinomas [10].

SÈVE et al. [11] demonstrated better outcome concerning PFS and $O S$ in patients treated with vinorelbine-based first-line chemotherapy having low protein expression of betatubulin III. Significantly improved survival was also observed in a similar gemcitabine-based treated patient group retrospectively analysed for RRM1-mRNA expression [20].

\section{DISCUSSION}

Individualised anticancer treatment based on the patient's biomarker profile is increasingly integrated in the management of various types of cancer, including NSCLC. The concept was initiated by anti-oestrogen treatment in breast cancer, based on tumour hormone receptor status, and has expanded greatly since. However, the road towards customised chemotherapy is not without obstacles, possibly owing to our evolving, but still not complete, knowledge of the complex subcellular pathways involved in malignancy. The purpose of this paper is to provide an overview of customised chemotherapy for patients with advanced NSCLC already implemented in daily practice, as well as the individualised treatment of tomorrow.

Based on the results of and CiUlEANu et al. [3] and SCAGLIOTTI et al. [4], nonsquamous advanced NSCLC patients are often treated with cisplatin/pemetrexed. An effective alternative in the same histopathological subgroup is bevacizumab plus PBDs, if available [13]. These RCTs were well powered, the exploratory analyses pre-specified and multicentre based. However, none of these were designed to answer histology questions. In addition, analysis of QoL, which is an extremely important parameter, was not explored. A tolerable toxicity profile is of great importance if clinicians are to customise treatment and the survival benefit should be weighed against a possible deterioration of QoL. This issue is often neglected in biomarker studies and has been explored and discussed in detail in a recent publication from our group [22].

Due to the large sample numbers, reproducibility and the fact that a variety of PBDs have largely the same effect on outcome [2], it seems justified that clinicians base their treatment according to these results, although they are retrospective and QoL investigation is warranted. Furthermore, strong evidence demonstrated that cisplatin combinations outperform carboplatin combinations in patients with nonsquamous NSCLC [2], thus making histopathology a useful tool in customised chemotherapy.

While there are many options for handling patients with adenocarcinoma/nonsquamous subtype, the situation concerning patients with squamous cell carcinomas is more limited. GeORGOUlias et al. [15] suggested in their large randomised chemotherapy trial that patients with nonadenocarcinoma subtype had a significantly better outcome when treated with cisplatin-taxane combination, which was supported by our research group [12]. The limitations of these studies are their unplanned retrospective nature and the fact that gemcitabine was part of a triplet regimen benefitting the patients with squamous cell carcinomas in our study. The overall picture, however, seems to suggest that taxane-based 
combinations is of value in this treatment resistant histological subgroup.

Histology-driven customisation is easy, cost-effective and is provided with the NSCLC diagnosis. The disadvantage is the pathologists' interobserver variation, the heterogenous nature of NSCLC tumour tissue as well as the occurrence of not otherwise specified (NOS) diagnosis, discussed in details in a recent review by ZALCMAN et al. [23]. Furthermore, the histopathological subtype is likely to be a surrogate marker for more complex subcellular mechanisms not yet fully uncovered, such as the thymidylate synthase hypothesis and pemetrexed resistance [24].

The expanding body of evidence concerning the prognostic and predictive value of EGFR status is comprehensive but incomplete. The EGFR pathway, involved in almost all aspects of cell growth (proliferation, angiogenesis and apoptosis, etc.), has been explored by three main methods: protein expression by using immunohistochemistry (IHC), gene amplification using fluorescence in situ hybridisation (FISH) and mutation analysis using direct sequencing or the PCR-based amplification refractory mutation system (ARMS) to reveal the most common types (deletion of exon 19 and exon 21 L858R mutation).

The BR21-study could demonstrate a positive predictive effect of EGFR IHC positivity on OS [18], a finding supported by the ISEL (Iressa Survival Evaluation in Lung Cancer) Study [17], including significant treatment interaction. However, when gefitinib was compared to docetaxel in the INTEREST (Iressa NSCLC Trial Evaluating Response and Survival Versus Taxotere) Study [16], there was no difference. All three studies demonstrated that IHC-negative patients should not be treated with gefitinib. A common problem with these studies, in addition to their retrospective nature, was the fact that only around one-third of the patients had representative tissue for evaluation, indicating cautious interpretation. Accordingly, nothing certain can be concluded on the positive predictive value of EGFR IHC status and more research is warranted concerning antibody quality and cut-off values, etc.

The picture is similar concerning FISH+ for EGFR gene amplification. The ISEL Study [17] and BR21 Study [18] proved gefitinib and erlotinib, respectively, to be of value when compared to placebo. This observation could not be confirmed when gefitinib was compared to docetaxel in the INTEREST Study [16], perhaps due to crossover after treatment failure. This study found a difference in RR in favour of gefitinib, but the end-point is probably not as robust as PFS or OS when evaluating TKIs. Again, the negative predictive value for gefitinib in FISH-negative patients was demonstrated in all three studies.

In the dawning of the EGFR era, TKIs were observed to be more efficient in certain subgroups (Asians, females, adenocarcinomas and nonsmokers). It was later shown that these groups often harboured typical EGFR mutations that vary in prevalence from $\sim 10 \%$ in Caucasian populations to $\sim 40 \%$ in Asians. Hence, these subgroups were selected for the IPASS (Iressa Pan-Asia Study) Study [7], securing a population prevalence of $59.7 \%$ positive for EGFR mutation. In this randomised study, designed for noninferiority with PFS as primary end-point, gefitinib was proven to be superior to carboplatin/docetaxel in advanced NSCLC first-line with an impressive HR of 0.16 in patients positive for the mutation. This positive predictive value was later supported by the INTEREST Study [16], but only among 44 retrospectively analysed patients in a second-line setting. Both studies demonstrated inferior efficacy of gefitinib compared with chemotherapy in the wild-type patients. The findings in the WJTOG3405 Study [6] and the planned interim analysis from the NEJ002 Study [5] strongly support the predictive value of EGFR mutation to gefitinib compared with PBDs in selected, randomised populations with EGFR mutation-positive patients; highly significant HRs of 0.49 and 0.36 , respectively, were demonstrated. Adding to the evidence is the feasibility study by Rosell et al. [25], in which the Spanish Lung Cancer Group screened 2,105 patients for EGFR mutations and treated 217 mutation-positive patients with erlotinib. Striking results were demonstrated: 14 and 27 months in PFS and OS, respectively. It should be noted, however, that in the WJTOG3405 Study $\sim 40 \%$ of the cohort were post-operative recurrence patients and thus not chemotherapy naïve.

Based on these findings, patients with advanced NSCLC carrying an EGFR mutation should be treated up front with gefitinib emphasising the need for EGFR status screening. This analysis is expensive, relatively time consuming and will only benefit smaller patient fractions, but will improve their outcome and reduce toxicity. However, the specificity and sensitivity of the different kits available for EGFR mutation analysis remains an issue of controversy, and a number of less common mutations are not revealed, increasing the false-negative rate.

While histology and EGFR status already allows customisation of treatment in advanced NSCLC, other promising biomarkers await implementation into daily clinical practice due to different obstacles.

ERCC1, involved in the excision of platin-induced DNA lesions, was proven of predictive value by the landmark study of OLAUSSEN et al. [9].The group retrospectively demonstrated that downregulation (allowing cisplatin to exert its apoptosisinducing effect) of the protein resulted in a significant favourable disease-free survival in the adjuvant setting. These findings led to the study by the Spanish Lung Cancer Group [19], who demonstrated feasibility of customising chemotherapy after stratification according to ERCC1 status. Outcome, however, was only slightly improved. Our group has recently confirmed the predictive value of ERCC1 status in the advanced setting. Furthermore, we found that the survival benefit could be attributed to the subgroup of patients with adenocarcinomas, suggesting that the predictive value may be restricted by histopathology [10].

Increased expression of betatubulin III has been associated with resistance to mitotic spindle poisons, like vinorelbine and taxanes, as demonstrated by SÈVE et al. [11]. Other biomarkers proving to be of potential predictive value are RRM1 [20], KRAS [21], EML4-ALK [26] and others, but these are too far beyond the scope of this review to discuss in detail.

Taken together, the biomarkers of tomorrow are promising, but have yielded different and sometimes conflicting results. The reasons may be small and heterogenous sample sizes, retrospective analyses, nonrandomised patient populations, 
problems concerning tissue samples and different cut-off values applied, as well as the fact that recommendations on biomarker studies (REMARK) [27] are not always followed consistently. A more detailed discussion of the methodology problems can be viewed in detail in our recent publication concerning ERCC1 and histopathology [10], as well as the review by ARMOUR and WATKINS [28] concerning EGFR.

Although the concept of using biomarkers for customised treatment in NSCLC may appear simple and straightforward, providing evidence is not without its obstacles. Owing to the problems mentioned above, many studies have been negative, and there is a great need for collaboration and common guidelines in order to secure valid results for rapid clinical implementation. It is encouraging to witness that such platforms are now being established (e.g. the ESMO Translational Research Working Group).

\section{Conclusions}

Based on current evidence, tailored treatment in advanced NSCLC is already feasible and should be part of daily clinical practice based on histology and EGFR mutation status. Concerning potential biomarkers, optimisation of methodology and prospective validation are warranted.

\section{SUPPORT STATEMENT}

The Harboe Foundation (Glostrup, Denmark), Augustinus Foundation (Copenhagen, Denmark) and the Research Council of Rigshospitalet (Copenhagen, Denmark) supported this study.

\section{STATEMENT OF INTEREST}

A.C. Vilmar and J.B. Sorensen have received fees for speaking and reimbursement for attending symposiums from Eli Lilly and Company.

\section{REFERENCES}

1 Ferlay J, Parkin DM, Steliarova-Foucher E. Estimates of cancer incidence and mortality in Europe in 2008. Eur J Cancer 2010; 46: 765-781.

2 Ardizzoni A, Boni L, Tiseo M, et al. Cisplatin- versus carboplatinbased chemotherapy in first-line treatment of advanced non-smallcell lung cancer: an individual patient data meta-analysis. J Natl Cancer Inst 2007; 99: 847-857.

3 Ciuleanu T, Brodowicz T, Zielinski C, et al. Maintenance pemetrexed plus best supportive care versus placebo plus best supportive care for non-small-cell lung cancer: a randomised, double-blind, phase 3 study. Lancet 2009; 374: 1432-1440.

4 Scagliotti GV, Parikh P, von Pawel J, et al. Phase III study comparing cisplatin plus gemcitabine with cisplatin plus pemetrexed in chemotherapy-naive patients with advanced-stage nonsmall-cell lung cancer. J Clin Oncol 2008; 26: 3543-3551.

5 Inoue A, Kobayashi K, Maemondo M, et al. A randomized phase III study comparing gefitinib with carboplatin (CBDCA) plus paclitaxel (TXL) for the first-line treatment of non-small cell lung cancer (NSCLC) with sensitive EGFR mutations. Eur J Cancer Suppl 2009; 7: 6 .

6 Mitsudomi T, Morita S, Yatabe Y, et al. Gefitinib versus cisplatin plus docetaxel in patients with non-small-cell lung cancer harbouring mutations of the epidermal growth factor receptor (WJTOG3405): an open label, randomised phase 3 trial. Lancet Oncol 2010; 11: 121-128.

7 Mok TS, Wu YL, Thongprasert S, et al. Gefitinib or carboplatinpaclitaxel in pulmonary adenocarcinoma. $N$ Engl J Med 2009; 361: 947-957.
8 Azuma K, Komohara Y, Sasada T, et al. Excision repair crosscomplementation group 1 predicts progression-free and overall survival in non-small cell lung cancer patients treated with platinum-based chemotherapy. Cancer Sci 2007; 98: 1336-1343.

9 Olaussen KA, Dunant A, Fouret P, et al. DNA repair by ERCC1 in non-small-cell lung cancer and cisplatin-based adjuvant chemotherapy. N Engl J Med 2006; 355: 983-991.

10 Vilmar AC, Santoni-Rugiu E, Sorensen JB. ERCC1 and histopathology in advanced NSCLC patients randomized in a large multicenter phase III trial. Ann Oncol 2010; 21: 1817-1824.

11 Seve $P$, Isaac $S$, Tredan $O$, et al. Expression of class III $\beta$-tubulin is predictive of patient outcome in patients with non-small cell lung cancer receiving vinorelbine-based chemotherapy. Clin Cancer Res 2005; 11: 5481-5486.

12 Sorensen J, Hansen O, Vilmar A, et al. Prospective randomized phase III trial of triplet chemotherapy with paclitaxel + gemcitabine + cisplatin compared to standard doublet chemotherapy with vinorelbine + cisplatin in advanced non-small cell lung cancer. J Clin Oncol 2009; 27: Suppl., 15s.

13 Sandler A, Yi J, Hambleton J, et al. Treatment outcomes by tumour histology in Eastern Cooperative Group (ECOG) study of bevacizumab (BV) with paclitaxel/carboplatin (PC) for advanced non-small cell lung cancer. J Thorac Oncol 2008; 3: Suppl. 4, S283.

14 Peterson P, Park K, Fossella FV, et al. Is pemetrexed more effective in adenocarcinoma and large cell lung cancer than in squamous cell carcinoma? A retrospective analysis of a phase III trial of pemetrexed vs docetaxel in previously treated patients with advanced non-small cell lung cancer (NSCLC). 12th World Conference on Lung Cancer (Abstracts). J Thorac Oncol 2007; 2: S851.

15 Georgoulias V, Papadakis E, Alexopoulos A, et al. Platinum-based and non-platinum-based chemotherapy in advanced non-smallcell lung cancer: a randomised multicentre trial. Lancet 2001; 357: 1478-1484.

16 Douillard JY, Shepherd FA, Hirsh V, et al. Molecular predictors of outcome with gefitinib and docetaxel in previously treated nonsmall-cell lung cancer: data from the randomized phase III INTEREST trial. J Clin Oncol 2010; 28: 744-752.

17 Hirsch FR, Varella-Garcia M, Bunn PA Jr, et al. Molecular predictors of outcome with gefitinib in a phase III placebocontrolled study in advanced non-small-cell lung cancer. J Clin Oncol 2006; 24: 5034-5042.

18 Tsao MS, Sakurada A, Cutz JC, et al. Erlotinib in lung cancer molecular and clinical predictors of outcome. N Engl J Med 2005; 353: 133-144.

19 Cobo M, Isla D, Massuti B, et al. Customizing cisplatin based on quantitative excision repair cross-complementing $1 \mathrm{mRNA}$ expression: a phase III trial in non-small-cell lung cancer. J Clin Oncol 2007; 25: 2747-2754.

20 Ceppi P, Volante M, Novello S, et al. ERCC1 and RRM1 gene expressions but not EGFR are predictive of shorter survival in advanced non-small-cell lung cancer treated with cisplatin and gemcitabine. Ann Oncol 2006; 17: 1818-1825.

21 Eberhard DA, Johnson BE, Amler LC, et al. Mutations in the epidermal growth factor receptor and in KRAS are predictive and prognostic indicators in patients with non-small-cell lung cancer treated with chemotherapy alone and in combination with erlotinib. J Clin Oncol 2005; 23: 5900-5909.

22 Vilmar A, Santoni-Rugiu E, Sorensen JB. ERCC1, toxicity and quality of life in advanced NSCLC patients randomized in a large multicentre phase III trial. Eur J Cancer 2010; 46: 1554-1562.

23 Zalcman G, Bergot E, Lechapt E. Update on nonsmall cell lung cancer. Eur Respir Rev 2010; 117: 173-185.

24 Ozasa H, Oguri T, Uemura T, et al. Significance of thymidylate synthase for resistance to pemetrexed in lung cancer. Cancer Sci 2010; 101: 161-166. 
25 Rosell R, Moran M, Queralt C, et al. Screening for epidermal growth factor receptor mutations in lung cancer. $N$ Engl J Med 2009; 361: 958-967.

26 Shaw AT, Yeap BY, Mino-Kenudson M, et al. Clinical features and outcome of patients with non-small-cell lung cancer who harbor EML4-ALK. J Clin Oncol 2009; 27: 4247-4253.
27 McShane LM, Altman DG, Sauerbrei W, et al. Reporting recommendations for tumour marker prognostic studies (REMARK). J Natl Cancer Inst 2005; 97: 1180-1184.

28 Armour AA, Watkins CL. The challenge of targeting EGFR: experience with gefitinib in nonsmall cell lung cancer. Eur Respir Rev 2010; 117: 186-196. 